\title{
Correction to: Preface
}

\section{Albert Cohen ${ }^{1}$. Wolfgang Dahmen ${ }^{2} \cdot$ Hans Munthe-Kaas ${ }^{3} \cdot$ Martín Sombra $^{4}$. Agnes Szanto 5}

Published online: 19 October 2021

(c) SFoCM 2021

\section{Correction to:}

\section{Foundations of Computational Mathematics (2019) 19:963-964 https://doi.org/10.1007/s10208-019-09434-y}

In the preface for this special issue, the authors affiliations were omitted. They are:

Albert Cohen

Laboratoire Jacques-Louis Lions, Sorbonne Université, 4 Place Jussieu, 75005 Paris, France

\section{Wolfgang Dahmen}

Mathematics Department, University of South Carolina, 1523 Greene Street, Columbia, SC 29208, USA

Hans Munthe-Kaas

Department of Mathematics, University of Bergen, Postbox 7803, 5020 Bergen, Norway

Martín Sombra

Institució Catalana de Recerca i Estudis Avançats (ICREA), Passeig Lluís Companys 23, 08010 Barcelona, Spain

The original article can be found online at https://doi.org/10.1007/s10208-019-09434-y.

$\varangle \quad$ Hans Munthe-Kaas

jofocm@gmail.com

1 Laboratoire Jacques-Louis Lions, Sorbonne Université, 4 Place Jussieu, 75005 Paris, France

2 Mathematics Department, University of South Carolina, 1523 Greene Street, Columbia, SC 29208, USA

3 Department of Mathematics, University of Bergen, Postbox 7803, 5020 Bergen, Norway

4 Institució Catalana de Recerca i Estudis Avançats (ICREA), Passeig Lluís Companys 23, 08010 Barcelona, Spain

5 Department of Mathematics, North Carolina State University, Campus Box 8205, Raleigh, NC 27965, USA 


\section{Agnes Szanto}

Department of Mathematics, North Carolina State University, Campus Box 8205, Raleigh, NC, 27965, USA

Publisher's Note Springer Nature remains neutral with regard to jurisdictional claims in published maps and institutional affiliations. 